ARTICLE

DOI: $10.1038 / \mathrm{s} 41467-017-01583-4$

\title{
Room temperature magnetization switching in topological insulator-ferromagnet heterostructures by spin-orbit torques
}

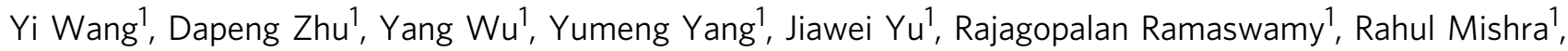
Shuyuan Shi',2, Mehrdad Elyasi ${ }^{1}$, Kie-Leong Teo ${ }^{1}$, Yihong Wu' ${ }^{1}$ \& Hyunsoo Yang ${ }^{1,2}$

Topological insulators with spin-momentum-locked topological surface states are expected to exhibit a giant spin-orbit torque in the topological insulator/ferromagnet systems. To date, the topological insulator spin-orbit torque-driven magnetization switching is solely reported in a $\mathrm{Cr}$-doped topological insulator at $1.9 \mathrm{~K}$. Here we directly show giant spin-orbit torquedriven magnetization switching in a $\mathrm{Bi}_{2} \mathrm{Se}_{3} / \mathrm{NiFe}$ heterostructure at room temperature captured using a magneto-optic Kerr effect microscope. We identify a large charge-to-spin conversion efficiency of $\sim 1-1.75$ in the thin $\mathrm{Bi}_{2} \mathrm{Se}_{3}$ films, where the topological surface states are dominant. In addition, we find the current density required for the magnetization switching is extremely low, $\sim 6 \times 10^{5} \mathrm{~A} \mathrm{~cm}^{-2}$, which is one to two orders of magnitude smaller than that with heavy metals. Our demonstration of room temperature magnetization switching of a conventional $3 d$ ferromagnet using $\mathrm{Bi}_{2} \mathrm{Se}_{3}$ may lead to potential innovations in topological insulator-based spintronic applications.

\footnotetext{
${ }^{1}$ Department of Electrical and Computer Engineering, National University of Singapore, Singapore 117576, Singapore. ${ }^{2}$ Centre for Advanced 2D Materials, National University of Singapore, 6 Science Drive 2, Singapore 117546, Singapore. Yi Wang and Dapeng Zhu contributed equally to this work.

Correspondence and requests for materials should be addressed to H.Y. (email: eleyang@nus.edu.sg)
} 
T he spin currents generated by charge currents via the spin Hall effect ${ }^{1-4}$ and/or Rashba-Edelstein effect ${ }^{5,6}$ can exert spin-orbit torques (SOTs) on the adjacent FM layer and result in the current-induced magnetization switching. A higher charge-to-spin conversion efficiency (referred as SOT efficiency) is crucial for the low-power dissipation SOT applications. Recently, the SOTs have been studied in topological insulators $(\mathrm{TIs})^{7-14}$, which are an emerging state of quantum matter possessing spin-momentum-locked topological surface states (TSS $)^{15-19}$. This exotic property is supposed to exhibit a large SOT efficiency, which is explored recently by the spin transport methods such as spin-torque ferromagnetic resonance $(\mathrm{ST}-\mathrm{FMR})^{7,8,13}$, spin pumping $9,10,14,18$, and spin tunneling spectroscopy ${ }^{19,20}$. However, in TIs such as $\mathrm{Bi}_{2} \mathrm{Se}_{3}$, the bulk states (BS) and two-dimensional electron gas (2DEG), which are typically present due to defects in the bulk and band bending at the surface $^{21,22}$, respectively, can lead to an inevitable contamination to the SOT effects from TSS. This is indicated by a wide range of the SOT efficiencies of $0.01-3.5$ reported in the $\mathrm{Bi}_{2} \mathrm{Se}_{3} /$ ferromagnet (FM) systems ${ }^{7-9,18}$. The roles of BS, 2DEG, and TSS on SOT efficiencies have not yet been clearly understood in details, which is critical for highly efficient SOT-driven magnetization switching using TIs.

To date, the magnetization switching induced by TI SOT is solely reported in a Cr-doped TI at a very low temperature $(1.9 \mathrm{~K})$ with an external magnetic field ${ }^{11}$, and the SOT-induced magnetization switching in a TI/3d FM heterostructure at room temperature is highly desired for applications. Here we obtain a TSS dominated SOT effect in 5-8 quintuple layers (QL) of $\mathrm{Bi}_{2} \mathrm{Se}_{3}$ films, exhibiting a large SOT efficiency of $\sim 1-1.75$ at room temperature using ST-FMR measurements. By taking advantage of the high efficiency, we image the SOT-induced magnetization switching by a magneto-optic Kerr effect (MOKE) microscope in the $\mathrm{Bi}_{2} \mathrm{Se}_{3} / \mathrm{NiFe}(\mathrm{Py})$ heterostructures at room temperature after injecting a pulsed dc current. The required current density for
SOT switching is extremely low and is one to two orders of magnitude smaller than that with heavy metals ${ }^{23-25}$. Our results suggest that TI/FM heterostructure could be a potential candidate for room temperature spintronic devices with ultralow-power dissipation.

\section{Results}

$\mathrm{Bi}_{2} \mathrm{Se}_{3}$ growth and film characterization. High-quality $\mathrm{Bi}_{2} \mathrm{Se}_{3}$ films ranging from 5 to $20 \mathrm{QL}(1 \mathrm{QL} \approx 1 \mathrm{~nm})$ were grown on $\mathrm{Al}_{2} \mathrm{O}_{3}$ (0001) substrates using molecular beam epitaxy (MBE) technique (see Methods). Figure 1a shows the atomic-force microscopy (AFM) image of a representative $10-\mathrm{QL} \mathrm{Bi}_{2} \mathrm{Se}_{3}$ film, indicating a smooth surface and high film quality. From the fourprobe and Hall measurements at room temperature, we find that the resistivity ( $\left.\rho_{\text {Bise }}\right)$ is $\sim 1,000 \mu \Omega \mathrm{cm}$ at large thicknesses (15 and $20 \mathrm{QL}$ ), increases at $10 \mathrm{QL}$ and becomes $\sim 4,117 \mu \Omega \mathrm{cm}$ at $5 \mathrm{QL}$, as shown in Fig. 1b. Moreover, the sheet resistance shows a similar trend as $\rho_{\text {BiSe }}$ (Supplementary Note 2 and Supplementary Fig. 2). The sheet carrier concentration $\left(n_{2 \mathrm{D}}\right)$ shows an opposite trend, decreasing from $\sim 6 \times 10^{13} \mathrm{~cm}^{-2}$ at 20 QL to $\sim 3.8 \times 10^{13} \mathrm{~cm}^{-2}$ below 8 QL. This behavior suggests a small contribution of BS and 2DEG to electrical transport properties in the thin $\mathrm{Bi}_{2} \mathrm{Se}_{3}$ cases, as we discuss later. We also characterize $\mathrm{Bi}_{2} \mathrm{Se}_{3}$ thickness $\left(t_{\text {BiSe }}\right)$-dependent $\rho_{\text {BiSe }}$ (Fig. 1c) and $n_{2 \mathrm{D}}$ (Fig. 1d) at different temperatures. Our $\mathrm{Bi}_{2} \mathrm{Se}_{3}$ films show a typical metallic behavior similar to previous reports ${ }^{8,26}$.

ST-FMR measurements. Figure 2a shows the schematic diagram of the ST-FMR measurement (see Methods section), an effective technique to evaluate the SOT efficiency ${ }^{7,27}$. The ST-FMR devices consist of $\mathrm{Bi}_{2} \mathrm{Se}_{3} \quad\left(t_{\mathrm{BiSe}}\right) / \mathrm{Co}_{40} \mathrm{Fe}_{40} \mathrm{~B}_{20} \quad(\mathrm{CFB}, 7 \mathrm{~nm})$ bilayers. Figure $2 \mathrm{~b}$ illustrates the current-induced spin polarization and magnetization dynamics in $\mathrm{Bi}_{2} \mathrm{Se}_{3} / \mathrm{CFB}$ bilayers. As an in-plane $\mathrm{rf}$ current $\left(I_{\mathrm{RF}}\right)$ flows in the $\mathrm{Bi}_{2} \mathrm{Se}_{3}$ layer, non-equilibrium spins are
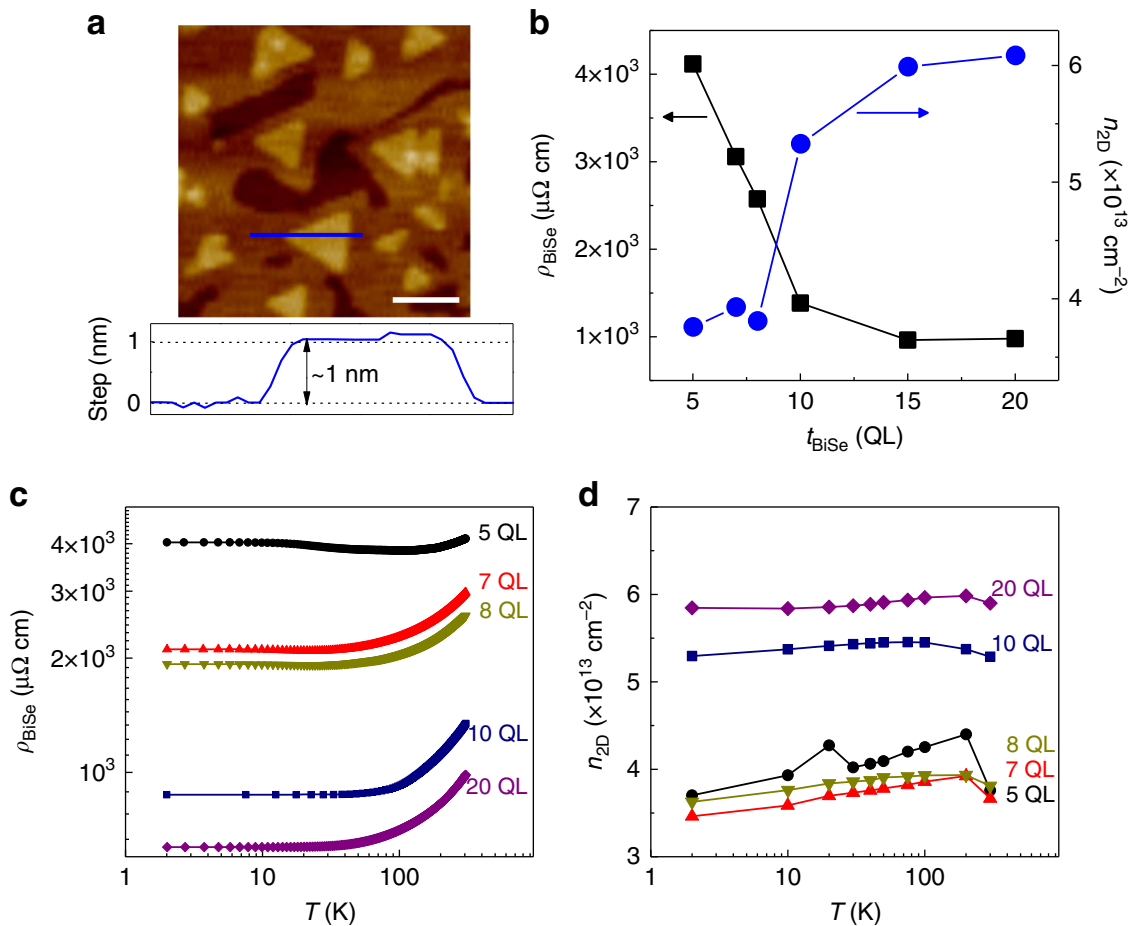

Fig. $1 \mathrm{Bi}_{2} \mathrm{Se}_{3}$ film properties. a AFM image of a 10-QL Bi $\mathrm{Se}_{3}$ film with a roughness of $\sim 0.5 \mathrm{~nm} .1 \mathrm{QL}(\approx 1 \mathrm{~nm})$ step of crystal terrace along the blue line is clearly observed. The white scale bar is $100 \mathrm{~nm}$. b Bi $\mathrm{Se}_{3}$ thickness-dependent resistivity, $\rho_{\mathrm{Bise}}$, and sheet carrier concentration, $n_{2 \mathrm{D}}$, in $\mathrm{Bi}_{2} \mathrm{Se}_{3}$ at room temperature. $\mathbf{c}, \mathbf{d} \rho_{\text {Bise }}$ and $n_{2 \mathrm{D}}$ as a function of temperature for $\mathrm{Bi}_{2} \mathrm{Se}_{3}$ films with different thicknesses, respectively 

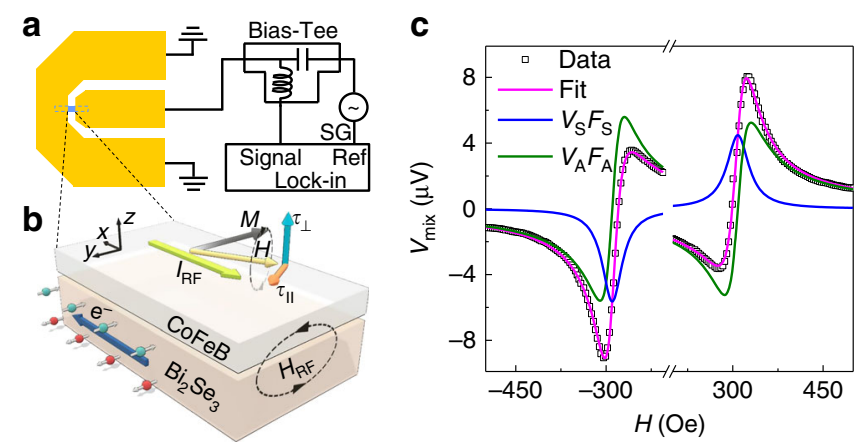

Fig. 2 ST-FMR measurements and signals with fits. a Schematic diagram of the ST-FMR measurement setup, illustrating a ST-FMR device and the measurement circuit. An rf current from a signal generator (SG) is injected into the ST-FMR devices via a bias-tee. b Illustration of spin momentum locking and SOT-induced magnetization dynamics in the ST-FMR

measurements. The big blue arrow denotes the electron moving direction (opposite to $I_{\mathrm{RF}}$ direction). The arrows with green and red balls denote the spin angular momentum generated at top and bottom surfaces of $\mathrm{Bi}_{2} \mathrm{Se}_{3}$, respectively. c A typical ST-FMR signal (open symbols) from a $\mathrm{Bi}_{2} \mathrm{Se}_{3} 20$ $\mathrm{QL} / \mathrm{CFB} 7 \mathrm{~nm}$ device at $6 \mathrm{GHz}$ with fits (solid lines), where the blue and green lines represent the symmetric Lorentzian $\left(V_{S} F_{S}\right)$ and antisymmetric Lorentzian $\left(V_{\mathrm{A}} F_{\mathrm{A}}\right)$ components, respectively

generated at the $\mathrm{Bi}_{2} \mathrm{Se}_{3}$ surfaces denoted by the arrows with green and red balls. These spins from $\mathrm{Bi}_{2} \mathrm{Se}_{3}$ top surface diffuse into $\mathrm{CFB}$ and exert oscillating damping-like torque $\left(\tau_{\mathrm{DL}}\right)$ and/or a field-like torque $\left(\tau_{\mathrm{FL}}\right)$ on the magnetization. These torques together with rf current-induced Oersted field $\left(H_{\mathrm{RF}}\right)$ torque $\left(\tau_{\mathrm{Oe}}\right)$ trigger the precession of CFB magnetization and an oscillation of the anisotropic magnetoresistance with the same frequency as $I_{\mathrm{RF}}$. Consequently, a mixing dc voltage $V_{\text {mix }}$ (i.e., ST-FMR signal) is produced across the ST-FMR device $7,8,27,28$.

Figure 2c shows typical ST-FMR signals $V_{\text {mix }}$ (open symbols), which are fitted by $V_{\text {mix }}=V_{\mathrm{S}} F_{\mathrm{S}}+V_{\mathrm{A}} F_{\mathrm{A}}$, where $F_{\mathrm{S}}$ and $F_{\mathrm{A}}$ are symmetric and antisymmetric Lorentzian functions, respectively. The amplitudes of symmetric $\left(V_{\mathrm{S}}\right)$ and antisymmetric component $\left(V_{\mathrm{A}}\right)$ are attributed to $\tau_{\mathrm{DL}}$ and $\tau_{\mathrm{FL}}+\tau_{\mathrm{Oe}}$, respectively ${ }^{7,27}$. By adopting the established analysis $\operatorname{method}^{7,8}$, the SOT efficiency $\left(\theta_{\mathrm{TI}}=J_{\mathrm{S}} / J_{\mathrm{C}}\right)$ can be evaluated from only $V_{\mathrm{S}}$ (Supplementary Note 3 ), where $J_{\mathrm{S}}$ is the spin current density at the $\mathrm{Bi}_{2} \mathrm{Se}_{3} / \mathrm{CFB}$ interface and $J_{\mathrm{C}}\left(\mathrm{A} \mathrm{cm}^{-2}\right)$ is the uniform charge current density in the $\mathrm{Bi}_{2} \mathrm{Se}_{3}$ layer. Similar measurements and analyses are further performed on devices with various $t_{\text {BiSe }}$ spanning 5-20 QL. Figure 3 shows $\theta_{\mathrm{TI}}$ vs. $t_{\mathrm{BiSe}}$ at room temperature. Each data point is averaged from three devices, which show a similar behavior. Specifically, $\theta_{\mathrm{TI}}$ shows a constant value of $\sim 0.3$ for 15 and $20 \mathrm{QL}$ devices, and starts to increase below $10 \mathrm{QL}$, reaching a maximum of $\sim 1.75$ at $5 \mathrm{QL}$. $\theta_{\mathrm{TI}}$ in thinner films increases $>5$ times compared to that in thicker $\mathrm{Bi}_{2} \mathrm{Se}_{3}$ devices. From the line shape of the ST-FMR signals and the positive sign of $\theta_{\mathrm{TI}}$ at different $t_{\mathrm{BiSe}}$, we confirm that the direction of in-plane spin polarization $\left(S_{\|}\right)$at the interface of $\mathrm{Bi}_{2} \mathrm{Se}_{3}$ and CFB is in line with TSS where $S_{\|}$is locked at right angles to the electron momentum ${ }^{7,8,29-31}$.

SOT efficiency vs. $\mathrm{Bi}_{2} \mathrm{Se}_{3}$ thickness and the role of TSS. Recent experimental and theoretical works ${ }^{21,32}$ indicate that as the thickness of $\mathrm{Bi}_{2} \mathrm{Se}_{3}$ decreases to several QLs, BS shrink significantly and finally disappear. In addition, the surface 2DEG bands are gradually quantized into discrete subbands enclosed by the linear TSS bands due to quantum confinement effects (Supplementary Fig. 3 ). Since the thickness of a TSS ( $\left.t_{\mathrm{TSS}}\right)$ and $2 \mathrm{DEG}$ $\left(t_{2 \mathrm{DEG}}\right)$ in $\mathrm{Bi}_{2} \mathrm{Se}_{3}$ are reported to be $\sim 1 \mathrm{~nm}^{21,26,32,33}$ and $\sim 4 \mathrm{~nm}^{21,26,32}$, respectively, negligible BS are expected when the

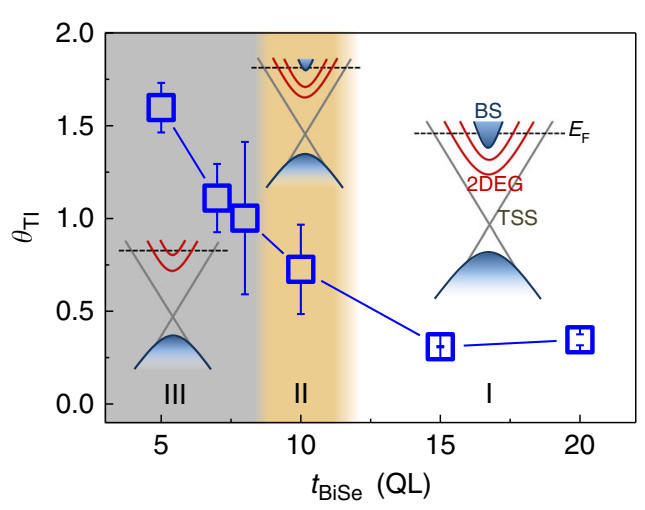

Fig. 3 The SOT efficiency $\left(\theta_{\mathrm{TI}}\right)$ as a function of $\mathrm{Bi}_{2} \mathrm{Se}_{3}$ thickness $\left(t_{\mathrm{BiSe}}\right)$ at room temperature. Each $\theta_{\mathrm{TI}}$ represents the averaged value from three devices. The error bars are the standard deviation. Region I, II, and III denoted by different colors represent the charge-to-spin conversion dominated by different mechanisms. The inset shows the schematic of the band structure for each region

$\mathrm{Bi}_{2} \mathrm{Se}_{3}$ thickness is $<8 \mathrm{QL}$. Accordingly, we discuss the transports in three regions (I, II, and III, denoted by different colors) in Fig. 3. In region $\mathrm{I}\left(t_{\mathrm{BiSe}}>10 \mathrm{QL}\right)$, there are considerable BS and 2DEG contributions to the transport, which could dilute the $\mathrm{TSS}^{8}$, resulting in a small $\theta_{\mathrm{TI}}$. In region II $(\sim 10 \mathrm{QL}), \mathrm{BS}$ start to shrink, leading to a slight increase of $\theta_{\mathrm{TI}}$. In region III $\left(t_{\mathrm{BiSe}} \leq 8 \mathrm{QL}\right)$, the BS disappear and the contribution from the 2DEG decreases as we discuss later. On the other hand, due to the lack of inversion symmetry in our devices, Rashba splitting states in 2DEG subbands can give rise to $S_{\|}$. However, the accumulated spins due to the Rashba states are expected to have an opposite helicity (i.e., negative $\theta_{\mathrm{TI}}$ ) compared to the TSS ${ }^{21,34-36}$. Since $\theta_{\mathrm{TI}}$ always shows positive values in all our devices, we conclude that the TSS dominated SOT is the main contribution to the large enhancement of $\theta_{\mathrm{TI}}$ in region III.

To further confirm that the TSS dominate SOT in region III (5-8 QL), we establish a model to quantify the carrier concentration in TSS ( $\left.n_{\text {TSS }}\right), 2$ DEG $\left(n_{2 \mathrm{DEG}}\right)$ and BS $\left(n_{2 \mathrm{D}-\mathrm{Bulk}}\right)$, as well as the corresponding current shunting effect due to BS and 2DEG (Supplementary Note 4 and Supplementary Fig. 3). As shown in Fig. 4 a, the $n_{2 \mathrm{DEG}}$ decreases significantly as $t_{\mathrm{BiSe}} \leq 8 \mathrm{QL}$, while $n_{\text {TSS }}$ shows a slight increase as $t_{\mathrm{BiSe}}$ decreases. This observation reproduces the inherent behaviors of TSS and 2DEG carriers measured in very thin $\mathrm{Bi}_{2} \mathrm{Se}_{3}$ films ${ }^{26}$. Moreover, the larger value of $n_{\text {TSS }}$ compared to $n_{2 \mathrm{DEG}}$ for $t_{\mathrm{BiSe}} \leq 8 \mathrm{QL}$ corroborates a TSS dominated transport in thin $\mathrm{Bi}_{2} \mathrm{Se}_{3}$ film region (Supplementary Note 4 and Supplementary Figs. 4-7). Figure $4 \mathrm{~b}$ shows the location of the Fermi level $\left(E_{\mathrm{F}}\right)$ relative to the Dirac point $\left(E_{\mathrm{DP}}\right)$ and Fermi vector $\left(k_{\mathrm{F}}\right)$, we find that $E_{\mathrm{F}}-E_{\mathrm{DP}}\left(k_{\mathrm{F}}\right)$ gradually increases from $\sim 403$ to $447 \mathrm{meV}$ (from $\sim 0.123$ to 0.135 $\AA^{-1}$ ) as $t_{\text {BiSe }}$ decreases, indicating that the DP slightly moves downwards to a larger binding energy which accounts for the weak increase of $n_{\mathrm{TSs}}$. The value of $E_{\mathrm{F}}-E_{\mathrm{DP}}\left(k_{\mathrm{F}}\right)$ and the DP movement are in line with previous ARPES measurements for $\mathrm{Bi}_{2} \mathrm{Se}_{3}$ films ${ }^{35,37-40}$. Figure $4 \mathrm{c}$ shows that the charge currents in the TSS on the top surface over the total currents flowing in $\mathrm{Bi}_{2} \mathrm{Se}_{3}\left(I_{\mathrm{TSS}} / I_{\text {total }}\right)$ increases from $\sim 0.2$ to 0.4 as $t_{\mathrm{BiSe}}$ decreases from 20 to $5 \mathrm{QL}$ (Supplementary Note 5 ), which again verifies that the TSS dominates the region III.

In addition, we estimate the "interface" SOT efficiency from TSS ( $\left.\lambda_{\text {TSS }}\right)$ by using an interface charge current density $J_{\mathrm{C}-\mathrm{TSS}}$ $\left(\mathrm{A} \mathrm{cm}^{-1}\right)$ in TSS (Supplementary Note 6 and Supplementary Figs. 8-11). As shown in Fig. 4 d, we find $\lambda_{\text {TSS }}$ is in the range of $\sim 0.38$ to $0.82 \mathrm{~nm}^{-1}$ when $t_{\mathrm{BiSe}} \leq 8 \mathrm{QL}$ at room temperature, which is consistent with recently reported interface SOT efficiency 

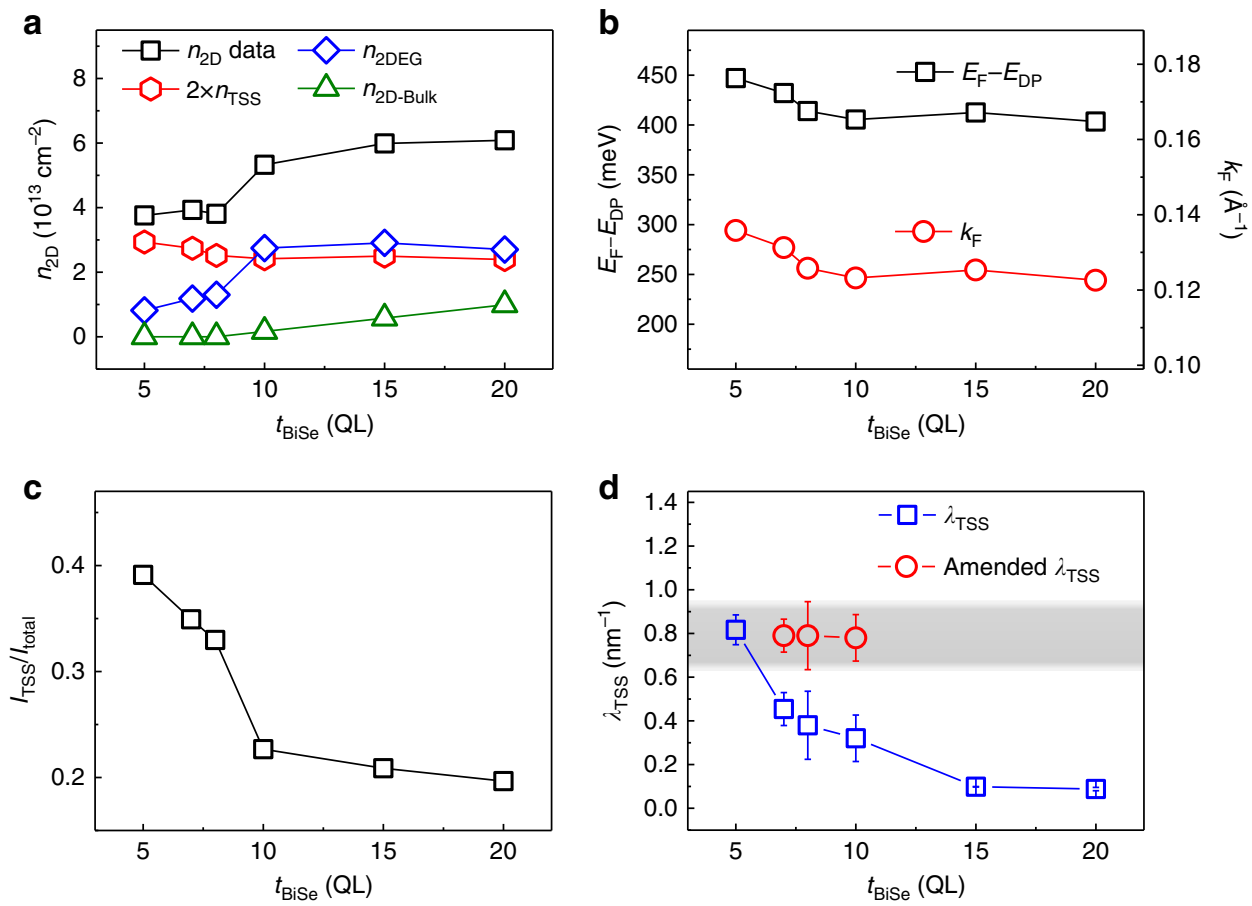

Fig. 4 Model analysis results and TSS dominated SOT in 5-8 QL Bi $\mathrm{Se}_{3}$. a Sheet carrier concentration of topological surface states (TSS), two-dimensional electron gas (2DEG) and bulk channels. b The location of the Fermi level relative to Dirac point $\left(E_{\mathrm{F}}-E_{\mathrm{DP}}\right)$ and Fermi vector $\left(k_{\mathrm{F}}\right)$ of TSS. $\mathbf{c}$ Ratio of charge currents in the TSS on the top surface and total currents in $\mathrm{Bi}_{2} \mathrm{Se}_{3}$. $\mathbf{d}$ Interface SOT efficiency, $\lambda_{\text {TSS }}$ (blue squares), as a function of $t_{\mathrm{BiSe}}$ at room temperature. The amended interface SOT efficiency from TSS after excluding the opposite $2 \mathrm{DEG}$ contribution is shown for 7, 8, and $10-\mathrm{QL} \mathrm{Bi} \mathrm{Se}_{3}$ in $\mathbf{d}$ with red circles. The factor of 2 in a arises due to the consideration of both the bottom and the top TSS in $\mathrm{Bi}_{2} \mathrm{Se}_{3}$. The error bars in $\mathbf{d}$ are the standard deviation from three devices at each $\mathrm{Bi}_{2} \mathrm{Se}_{3}$ thickness

values in $\left(\mathrm{Bi}_{1-x} \mathrm{Sb}_{x}\right)_{2} \mathrm{Te}_{3}{ }^{13}$. In principle, $\lambda_{\mathrm{TSS}}$ is inversely propotional to the Fermi velocity $V_{\mathrm{F}}$ and remains almost constant at different $t_{\mathrm{BiSe}}{ }^{13}$, however, a pronounced variation of $\lambda_{\mathrm{TSS}}$ is observed. This deviation unambiguously suggests that there is an opposite spin accumulation mechanism which cancels part of the spins generated by TSS in $\mathrm{Bi}_{2} \mathrm{Se}_{3}$. We attribute this to the Rashba states in $2 \mathrm{DEG}^{21,34-36}$. From the change of $\lambda_{\mathrm{TSS}}$, we can extract the interface SOT efficiency from $2 \mathrm{DEG}\left(\lambda_{2 \mathrm{DEG}}\right) \approx-0.4 \mathrm{~nm}^{-1}$ in the thin film regime (Supplementary Note 7). After excluding the 2DEG contribution, the amended interface SOT efficiency from TSS denoted by red circles in Fig. 4d, shows a constant value of $\sim 0.8 \mathrm{~nm}^{-1}$ for 7,8 and $10-\mathrm{QL} \mathrm{Bi}_{2} \mathrm{Se}_{3}$ devices, which is simliar to the value of $\lambda_{\mathrm{TSS}} \sim 0.82 \mathrm{~nm}^{-1}$ at $t_{\mathrm{BiSe}}=5 \mathrm{QL}$ (Supplementary Note 7 and Supplementary Figs. 8-11).

SOT-driven magnetization switching in $\mathrm{Bi}_{2} \mathrm{Se}_{3} / \mathrm{Py}$. The STFMR measurements and the above analysis reveal that the contribution of TSS is dominant in the thin $\mathrm{Bi}_{2} \mathrm{Se}_{3}$ films (5-8 QL), leading to a higher SOT efficiency at room temperature. Subsequently, we demonstrate the SOT-induced magnetization switching in $\mathrm{Bi}_{2} \mathrm{Se}_{3}(8 \mathrm{QL}) / \mathrm{Py}(6 \mathrm{~nm})$ heterostructures (see Methods section) at room temperature by applying a pulsed $\mathrm{dc}$ current $I$. The high-resolution scanning transmission electron microscope (STEM) image shows a clean and smooth interface between the $\mathrm{Bi}_{2} \mathrm{Se}_{3}$ and Py layer (see Supplementary Note 1 and Supplementary Fig. 1). In order to take advantage of the higher SOT efficiency and flow enough charge currents in the $\mathrm{Bi}_{2} \mathrm{Se}_{3}$ layer, an $8-\mathrm{QL} \mathrm{Bi}_{2} \mathrm{Se}_{3}$ is utilized. As depicted in Fig. 5a, the continuous Py layer is separated into five well-defined rectangles (yellow dashed boxes) and magnetically isolated by $\mathrm{Cu}$ bars. The magnetic easy axis of Py rectangles is along $\pm y$ directions due to the shape anisotropy. The magnetization direction of Py is collinear with the incoming spin directions (Fig. 2b) and thus the spins can directly switch the magnetization direction of Py without any external assisted magnetic field, which are captured by MOKE imaging measurements (see Methods section).

Figure 5a-e (top panel) show the SOT-driven magnetization switching by applying a pulsed $I$ along the $+x$-axis. At the beginning of this set of measurements, we first saturate the Py magnetization along the $+y$-axis by applying an in-plane external magnetic field $(H)$. Then, we remove $H$ and apply $I$ along the $+x$-axis to the device. When the current density in $\mathrm{Bi}_{2} \mathrm{Se}_{3}\left(J_{\mathrm{C}}\right)$ is zero, we capture the MOKE image as shown in Fig. 5a. The dark contrast represents the magnetization along the $+y$-axis, indicated by the white arrow. We find that as $J_{C}$ increases, the area of the switched magnetization with light contrast gradually expands (see Fig. 5b-d). Finally, the magnetization of all Py rectangles is switched to the $-y$-axis at $J_{\mathrm{C}}=5.7 \times 10^{5} \mathrm{~A} \mathrm{~cm}^{-2}$, which is indicated by the white arrow in Fig. 5e. Similarly, for the other set of measurements in Fig. $5 f-j$, we first initialize the Py magnetization along the $-y$-axis. Then we remove $H$ and apply $I$ of opposite polarity, i.e., along the $-x$-axis. As $J_{\mathrm{C}}$ increases, the Py magnetization switches from the $-y$ (Fig. 5 f, light contrast) to $+y$ axis (Fig. 5j, dark contrast) at $J_{\mathrm{C}}=6.2 \times 10^{5} \mathrm{~A} \mathrm{~cm}^{-2}$, exhibiting the opposite switching direction. The SOT-induced switching is reproducible in other devices (Supplementary Note 8 and Supplementary Fig. 12). We find that the current density required for the room temperature SOT-induced magnetization switching in $\mathrm{Bi}_{2} \mathrm{Se}_{3} / \mathrm{Py}$ is extremely low at $\sim 6 \times 10^{5} \mathrm{~A} \mathrm{~cm}^{-2}$, which is one to two orders of magnitude smaller than that with heavy metals ${ }^{23-25}$. Moreover, based on the conventional antidamping spin-torque-driven magnetization switching model ${ }^{24,41}$ with consideration of thermal fluctuation and reverse domain nucleation, we determine the SOT efficiency for $\mathrm{Bi}_{2} \mathrm{Se}_{3} / \mathrm{Py}$ to be $\sim 1.71$, which is in accord with the value from our ST-FMR measurements (Supplementary Note 9). This agreement further 


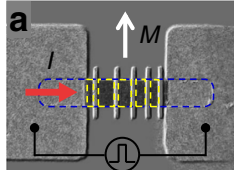

$J_{C}=0 \times 10^{5} \mathrm{~A} \mathrm{~cm}^{-2}$

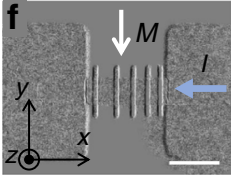

$J_{\mathrm{C}}=0 \times 10^{5} \mathrm{~A} \mathrm{~cm}^{-2}$

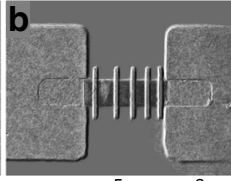

$3.3 \times 10^{5} \mathrm{~A} \mathrm{~cm}^{-2}$

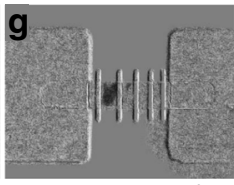

$3.8 \times 10^{5} \mathrm{~A} \mathrm{~cm}^{-2}$

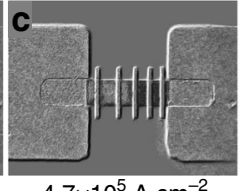

$4.7 \times 10^{5} \mathrm{~A} \mathrm{~cm}^{-2}$

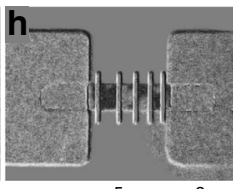

$5.2 \times 10^{5} \mathrm{~A} \mathrm{~cm}^{-2}$

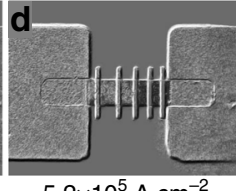

$5.2 \times 10^{5} \mathrm{~A} \mathrm{~cm}^{-2}$

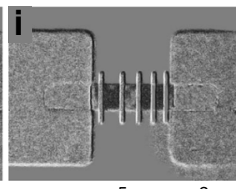

$5.7 \times 10^{5} \mathrm{~A} \mathrm{~cm}^{-2}$
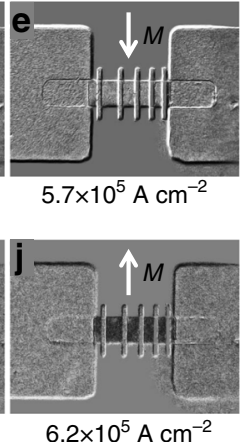

Fig. $5 \mathrm{MOKE}$ images of SOT-driven magnetization switching in $\mathrm{Bi}_{2} \mathrm{Se}_{3} / \mathrm{Py}$ at zero magnetic field and room temperature. a-e, MOKE images for SOT-driven magnetization switching by applying a pulsed dc current $I$ along the $+x$-axis with increasing the current density $J_{C}$ in the $\mathrm{Bi}_{2} \mathrm{Se}_{3}$ layer denoted underneath the corresponding image. The blue dashed rectangle in a represents the $12-\mu \mathrm{m}$ wide $\mathrm{Bi}_{2} \mathrm{Se}_{3} / \mathrm{Py}$ channel connected with two big contact pads. The yellow dashed boxes in a denote five small Py rectangles magnetically isolated by Cu bars. $\mathbf{f}-\mathbf{j}$, MOKE images for SOT-driven magnetization switching for I along $x$-axis. The dark (light) contrast shows the magnetization along $+y(-y)$-axis. The direction of magnetization is also indicated by the white arrows in $\mathbf{a}, \mathbf{e}, \mathbf{f}, \mathbf{j}$. The white scale bar is $20 \mu \mathrm{m}$

corroborates the excellent efficiency of TIs in spin generation and SOT-driven magnetization switching. Moreover, the robust SOTinduced magnetization switching is also observed in devices with a $\mathrm{Cu}$ or $\mathrm{NiO}$ insertion layer between the $\mathrm{Bi}_{2} \mathrm{Se}_{3}$ and Py layer (Supplementary Note 11 and Supplementary Figs. 14-16). From the control measurements, we find that neither the Joule heating nor the current-induced Oersted field could lead to the observed current-induced magnetization switching (Supplementary Notes 10 and 12, Supplementary Figs. 13 and 17).

\section{Discussion}

It has been suggested that interdiffusion may occur at the interface between TI and transition metals ${ }^{42,43}$, which can play a role in the TI SOT-induced magnetization switching despite maintaining the TSS ${ }^{43}$. The exact role of interdiffusion in the switching process, however, remains unclear, and suggests that future studies focused on the TI/FM interface are necessary.

The fundamental obstacle for high-density non-volatile applications of magnetic devices in a conventional spin torque scheme is the high critical switching current density, resulting in a large size of the current driving transistor. Utilizing the giant SOT effect in $\mathrm{Bi}_{2} \mathrm{Se}_{3}$, which can be grown in a wafer scale using MBE, we achieve a significantly low $J_{\mathrm{C}}$ to switch a conventional $3 d$ ferromagnet NiFe, which is widely utilized in industries, addressing an outstanding scalability issue in modern magnetic devices. Moreover, no requirement of an assistive magnetic field for our demonstrated magnetization switching scheme makes the TI/FM material systems easy to integrate into the established industrial technology for magnetic devices. Our above findings may bring this exotic newly discovered quantum matter from research activities to core ingredients in real spintronic applications.

\section{Methods}

Film growth and device fabrication. High-quality $\mathrm{Bi}_{2} \mathrm{Se}_{3}$ films ranging from 5 to 20 QLs are grown on $\mathrm{Al}_{2} \mathrm{O}_{3}$ (0001) substrates in a MBE system (MBC-1000-2C from ULVAC) with a base pressure $<1.5 \times 10^{-9}$ Torr, by using two-step deposition procedure $^{44,45}$. The sapphire substrates are first cleaned in acetone, isopropanol and de-ionized water, and subsequently annealed at $750{ }^{\circ} \mathrm{C}$ for $30 \mathrm{~min}$ in a vacuum after being transferred into the growth chamber. Elemental $\mathrm{Bi}(6 \mathrm{~N})$ and $\mathrm{Se}(5 \mathrm{~N})$ solid sources are evaporated from standard Knudsen cells under a $\mathrm{Se} / \mathrm{Bi}$ flux ratio of $\sim 20$. To reduce $\mathrm{Se}$ vacancies in $\mathrm{Bi}_{2} \mathrm{Se}_{3}$, initial 2-3 $\mathrm{QL} \mathrm{Bi}_{2} \mathrm{Se}_{3}$ are deposited at $150^{\circ}$ $\mathrm{C}$, and then the substrate temperature is ramped to $250^{\circ} \mathrm{C}$ at $5^{\circ} \mathrm{C} \mathrm{min}{ }^{-1}$ under Se flux for the second step growth. Our $\mathrm{Bi}_{2} \mathrm{Se}_{3}$ films have smooth surface with a roughness of $\sim 0.5 \mathrm{~nm}$ and show a clear terrace step of $\sim 1 \mathrm{~nm}(\approx 1 \mathrm{QL})$. The morphology indicates the high quality of our $\mathrm{Bi}_{2} \mathrm{Se}_{3}$ films. After the $\mathrm{Bi}_{2} \mathrm{Se}_{3}$ growth, the bare $\mathrm{Bi}_{2} \mathrm{Se}_{3}$ films are immediately transferred into a magnetron sputtering chamber via air in the standard cleanroom environment with a well-controlled levels of low humidity and constant temperature. The transfer time was strictly controlled under 5 min before pumping down the sputtering chamber. For the STFMR devices, a 7-nm thick $\mathrm{Co}_{40} \mathrm{Fe}_{40} \mathrm{~B}_{20}$ (CFB) is subsequently sputtered on the $\mathrm{Bi}_{2} \mathrm{Se}_{3}$ film with a low power of $60 \mathrm{~W}$ at room temperature with a base pressure of $\sim 3 \times 10^{-9}$ Torr. Finally, the $\mathrm{Bi}_{2} \mathrm{Se}_{3} / \mathrm{CFB}$ bilayer is protected by the sputtered $\mathrm{MgO}$ $(2 \mathrm{~nm}) / \mathrm{Al}_{2} \mathrm{O}_{3}(3 \mathrm{~nm})$ layer. For the MOKE imaging devices, the Py $(6 \mathrm{~nm}) / \mathrm{MgO}$ $(1 \mathrm{~nm}) / \mathrm{SiO}_{2}(4 \mathrm{~nm})$ stacks are subsequently sputtered onto the $\mathrm{Bi}_{2} \mathrm{Se}_{3}(8 \mathrm{QL})$ films with an in-situ magnetic field along the $y$-axis (i.e., perpendicular to the current channel, see Fig. $5 \mathrm{f}$ ) at room temperature with a base pressure of $\sim 3 \times 10^{-9}$ Torr. A very low sputtering power of $40 \mathrm{~W}$ is used for the Py deposition. Subsequently, five $2-\mu \mathrm{m}$ wide grooves on the Py layer are etched and backfilled with nonmagnetic metal $\mathrm{Cu}$, which divide the continuous Py layer into five rectangles and make them magnetically isolated. All devices are patterned by photolithography and ion milling.

ST-FMR measurements. The ST-FMR signals are detected by a lock-in amplifier. The frequencies and nominal power of the rf current $I_{\mathrm{RF}}$ are $6-9 \mathrm{GHz}$ and $15 \mathrm{dBm}$ respectively. An in-plane external magnetic field $(H)$ is applied at a fixed angle $\left(\theta_{\mathrm{H}}\right)$ of $35^{\circ}$ with respect to $I_{\mathrm{RF}}$.

MOKE imaging measurements. The Py magnetic easy anisotropy in the device is along the $\pm y$-axis (Fig. $5 \mathrm{f}$ ) due to the shape anisotropy. This allows us to capture the magnetization switching after pulsed dc current is off, where there is no current-induced spurious effects in the MOKE images. For the MOKE imaging measurements, we first saturate the Py magnetization along the $+y$ or $-y$-axis with an in-plane external magnetic field $H$, then we remove $H$ and apply a pulsed dc current $(500 \mu$ s pulse width) to observe the magnetization switching using MOKE microscope.

Data availability. The data that support the findings of this study are available from the corresponding author on request.

Received: 6 April 2017 Accepted: 29 September 2017

Published online: 08 November 2017

\section{References}

1. Hirsch, J. E. Spin Hall effect. Phys. Rev. Lett. 83, 1834-1837 (1999).

2. Zhang, S. Spin Hall effect in the presence of spin diffusion. Phys. Rev. Lett. 85 , 393-396 (2000).

3. Hoffmann, A. Spin Hall effects in metals. IEEE. Trans. Magn. 49, 5172-5193 (2013).

4. Sinova, J., Valenzuela, S. O., Wunderlich, J., Back, C. H. \& Jungwirth, T. Spin Hall effects. Rev. Mod. Phys. 87, 1213-1260 (2015).

5. Bychkov, Y. A. \& Rashba, E. Properties of a 2D electron gas with lifted spectral degeneracy. JETP lett. 39, 78 (1984).

6. Edelstein, V. M. Spin polarization of conduction electrons induced by electric current in two-dimensional asymmetric electron systems. Solid State Commun. 73, 233-235 (1990).

7. Mellnik, A. et al. Spin-transfer torque generated by a topological insulator. Nature 511, 449-451 (2014). 
8. Wang, Y. et al. Topological surface states originated spin-orbit torques in $\mathrm{Bi}_{2} \mathrm{Se}_{3}$. Phys. Rev. Lett. 114, 257202 (2015).

9. Deorani, P. et al. Observation of inverse spin Hall effect in bismuth selenide. Phys. Rev. B 90, 094403 (2014).

10. Shiomi, Y. et al. Spin-electricity conversion induced by spin injection into topological insulators. Phys. Rev. Lett. 113, 196601 (2014).

11. Fan, Y. et al. Magnetization switching through giant spin-orbit torque in a magnetically doped topological insulator heterostructure. Nat. Mater. 13, 699-704 (2014).

12. Rojas-Sánchez, J. C. et al. Spin to charge conversion at room temperature by spin pumping into a new type of topological insulator: $\alpha$-Sn films. Phys. Rev. Lett. 116, 096602 (2016).

13. Kondou, K. et al. Fermi-level-dependent charge-to-spin current conversion by Dirac surface states of topological insulators. Nat. Phys. 12, 1027-1031 (2016).

14. Wang, H. et al. Surface-state-dominated spin-charge current conversion in topological-insulator-ferromagnetic-insulator heterostructures. Phys. Rev. Lett. 117, 076601 (2016).

15. Hasan, M. Z. \& Kane, C. L. Colloquium: topological insulators. Rev. Mod. Phys. 82, 3045-3067 (2010).

16. Qi, X.-L. \& Zhang, S.-C. Topological insulators and superconductors. Rev. Mod. Phys. 83, 1057-1110 (2011).

17. Moore, J. E. The birth of topological insulators. Nature 464, 194-198 (2010).

18. Jamali, M. et al. Giant spin pumping and inverse Spin Hall effect in the presence of surface and bulk spin-orbit coupling of topological insulator $\mathrm{Bi}_{2} \mathrm{Se}_{3}$. Nano. Lett. 15, 7126-7132 (2015).

19. Liu, L. et al. Spin-polarized tunneling study of spin-momentum locking in topological insulators. Phys. Rev. B 91, 235437 (2015).

20. Lee, J. S., Richardella, A., Hickey, D. R., Mkhoyan, K. A. \& Samarth, N. Mapping the chemical potential dependence of current-induced spin polarization in a topological insulator. Phys. Rev. B 92, 155312 (2015).

21. Bahramy, M. S. et al. Emergent quantum confinement at topological insulator surfaces. Nat. Commun. 3, 1159 (2012).

22. Bianchi, M. et al. Coexistence of the topological state and a two-dimensional electron gas on the surface of $\mathrm{Bi}_{2} \mathrm{Se}_{3}$. Nat. Commun. 1, 128 (2010).

23. Miron, I. M. et al. Perpendicular switching of a single ferromagnetic layer induced by in-plane current injection. Nature 476, 189-193 (2011).

24. Liu, L. et al. Spin-torque switching with the giant Spin Hall effect of tantalum. Science 336, 555 (2012).

25. Liu, L., Lee, O. J., Gudmundsen, T. J., Ralph, D. C. \& Buhrman, R. A. Currentinduced switching of perpendicularly magnetized magnetic layers using spin torque from the Spin Hall effect. Phys. Rev. Lett. 109, 096602 (2012).

26. Bansal, N., Kim, Y. S., Brahlek, M., Edrey, E. \& Oh, S. Thickness-independent transport Channels in topological insulator $\mathrm{Bi}_{2} \mathrm{Se}_{3}$ thin films. Phys. Rev. Lett. 109, 116804 (2012).

27. Liu, L., Moriyama, T., Ralph, D. C. \& Buhrman, R. A. Spin-torque ferromagnetic resonance induced by the Spin Hall effect. Phys. Rev. Lett. 106, 036601 (2011)

28. Wang, Y., Deorani, P., Qiu, X., Kwon, J. H. \& Yang, H. Determination of intrinsic spin Hall angle in Pt. Appl. Phys. Lett. 105, 152412 (2014).

29. Hsieh, D. et al. Observation of unconventional quantum spin textures in topological insulators. Science 323, 919 (2009).

30. Pan, Z. H. et al. Electronic structure of the topological insulator $\mathrm{Bi}_{2} \mathrm{Se}_{3}$ using angle-resolved photoemission spectroscopy: evidence for a nearly full surface spin polarization. Phys. Rev. Lett. 106, 257004 (2011).

31. Jozwiak, C. et al. Photoelectron spin-flipping and texture manipulation in a topological insulator. Nat. Phys. 9, 293-298 (2013).

32. Park, B. C. et al. Terahertz single conductance quantum and topological phase transitions in topological insulator $\mathrm{Bi}_{2} \mathrm{Se}_{3}$ ultrathin films. Nat. Commun. 6, 6552 (2015).

33. Linder, J., Yokoyama, T. \& Sudbø, A. Anomalous finite size effects on surface states in the topological insulator $\mathrm{Bi}_{2} \mathrm{Se}_{3}$. Phys. Rev. B 80, 205401 (2009).

34. Wray, L. A. et al. A topological insulator surface under strong Coulomb, magnetic and disorder perturbations. Nat. Phys. 7, 32-37 (2011).

35. King, P. D. C. et al. Large tunable rashba spin splitting of a two-dimensional electron gas in $\mathrm{Bi}_{2} \mathrm{Se}_{3}$. Phys. Rev. Lett. 107, 096802 (2011).
36. Fischer, M. H., Vaezi, A., Manchon, A. \& Kim, E.-A. Large spin torque in topological insulator/ferromagnetic metal bilayers. Phys. Rev. B 93, 125303 (2013).

37. Xia, Y. et al. Observation of a large-gap topological-insulator class with a single Dirac cone on the surface. Nat. Phys. 5, 398-402 (2009).

38. Zhang, Y. et al. Crossover of the three-dimensional topological insulator $\mathrm{Bi}_{2} \mathrm{Se}_{3}$ to the two-dimensional limit. Nat. Phys. 6, 584-588 (2010).

39. Kuroda, K. et al. Hexagonally deformed fermi surface of the 3D topological insulator $\mathrm{Bi}_{2} \mathrm{Se}_{3}$. Phys. Rev. Lett. 105, 076802 (2010).

40. Neupane, $M$. et al. Observation of quantum-tunnelling-modulated spin texture in ultrathin topological insulator $\mathrm{Bi}_{2} \mathrm{Se}_{3}$ films. Nat. Commun. 5, 3841 (2014).

41. Fukami, S., Anekawa, T., Zhang, C. \& Ohno, H. A spin-orbit torque switching scheme with collinear magnetic easy axis and current configuration. Nat. Nanotech. 11, 621-625 (2016).

42. Shaughnessy, M., Bartelt, N., Zimmerman, J. \& Sugar, J. Energetics and diffusion of gold in bismuth telluride-based thermoelectric compounds. J. Appl. Phys. 115, 063705 (2014)

43. Eich, A. et al. Absence of superconductivity in ultrathin layers of FeSe synthesized on a topological insulator. Phys. Rev. B 94, 125437 (2016).

44. Li, H. et al. The van der Waals epitaxy of $\mathrm{Bi}_{2} \mathrm{Se}_{3}$ on the vicinal $\mathrm{Si}$ (111) surface: an approach for preparing high-quality thin films of a topological insulator. New. J. Phys. 12, 103038 (2010).

45. Bansal, N. et al. Epitaxial growth of topological insulator $\mathrm{Bi}_{2} \mathrm{Se}_{3}$ film on $\mathrm{Si}$ (111) with atomically sharp interface. Thin Solid Films 520, 224-229 (2011).

\section{Acknowledgements}

This work was supported by the A`STAR’s Pharos Programme on Topological Insulators.

\section{Author contributions}

Y.W. and H.Y.: Conceived and designed the research. D.Z., Yi.W. and K.-L.T.: Carried out thin film growth and characterization. Y.W. and D.Z.: Carried out ST-FMR sample preparation and measurements with inputs from R.M., R.R. and S.S. Y.W. and Y.Y.: Fabricated the samples for MOKE measurements. Y.W. and Y.Wu.: Performed MOKE imaging measurements. Y.Wu.: Prepared the MOKE setup. Y.W. and M.E.: Performed model the analysis. Y.W., D.Z., J.Y., R.R. and H.Y.: Prepared the figures and manuscript. All authors discussed the data and the results. H.Y.: Supervised the project.

\section{Additional information}

Supplementary Information accompanies this paper at doi:10.1038/s41467-017-01583-4

Competing interests: The authors declare no competing financial interests.

Reprints and permission information is available online at http://npg.nature.com/ reprintsandpermissions/

Publisher's note: Springer Nature remains neutral with regard to jurisdictional claims in published maps and institutional affiliations.

\section{(i)}

Open Access This article is licensed under a Creative Commons Attribution 4.0 International License, which permits use, sharing, adaptation, distribution and reproduction in any medium or format, as long as you give appropriate credit to the original author(s) and the source, provide a link to the Creative Commons license, and indicate if changes were made. The images or other third party material in this article are included in the article's Creative Commons license, unless indicated otherwise in a credit line to the material. If material is not included in the article's Creative Commons license and your intended use is not permitted by statutory regulation or exceeds the permitted use, you will need to obtain permission directly from the copyright holder. To view a copy of this license, visit http://creativecommons.org/ licenses/by/4.0/.

(c) The Author(s) 2017 